\title{
Photon trains and lasing: The periodically pumped quantum dot
}

\author{
Christian Wiele* and Fritz Haake \\ Fachbereich Physik der Universität-GH Essen, D-45117 Essen, Germany
}

Carsten Rocke and Achim Wixforth

Sektion Physik der Ludwig-Maximilians-Universität, Geschwister-Scholl-Platz, 1, D-80539 München, Germany

(Received 24 March 1998)

\begin{abstract}
We propose to pump semiconductor quantum dots with surface acoustic waves that deliver an alternating periodic sequence of electrons and holes. In combination with a good optical cavity such regular pumping could entail antibunching and sub-Poissonian photon statistics. In the bad-cavity limit a train of equally spaced photons would arise. [S1050-2947(98)51410-2]

PACS number(s): 42.50.Ct, 42.50.Lc, 42.55.Sa, 77.65.Dq
\end{abstract}

\section{INTRODUCTION}

Semiconductor quantum dots have an interesting potential for quantum-optical applications. The growth of dots with transition frequencies in the optical range is very well controlled [1]. Such a zero-dimensional system leads to much higher gain than bulk or two-dimensional quantum-well structures, as shown theoretically as well as experimentally [1-4]. Dots as active media in semiconductor lasers have already been established, and even lasing of a single dot in a semiconductor microcavity can be achieved [5-7]. From a theoretical point of view, the discrete states allow us to treat dots much like atoms. This makes for a much simpler situation than, for example, the continua of states in quantum wells. Furthermore, the semiconductor samples are small compared to atomic beams or even clouds of trapped atoms.

If a dot is to be operated as a low-noise light source it had better be pumped in an as regular a manner as possible. Imamoḡlu and Yamamoto's scheme of a single-photon turnstile device [8] driven by an alternating voltage source is one method for a regularized pump. A surface acoustic wave (SAW) as well could periodically deliver electrons and holes at a well localized array of dots or even a single dot [9-11].

\section{THE DEVICE}

To briefly explain our concept, let us consider a semiconductor quantum well surrounded by a piezoelectric material with an interdigital transducer (IDT) on top of the crystal (Fig. 1). A mechanical SAW is generated by applying a high-frequency signal to the IDT. The fundamental acoustic wavelength $\lambda_{0}$ and the frequency $f_{0}=v / \lambda_{0}$ are established by the interdigital electrode spacing, where $v$ is the sound velocity of the crystal. With $\lambda_{0} \sim 1-3 \mu \mathrm{m}$ and $v$ $\sim 3 \mathrm{~km} \mathrm{~s}^{-1}$, frequencies in the $\mathrm{GHz}$ range are achievable. The acoustic wave is accompanied by a piezoelectric field that gives an additional potential for electrons and holes and so periodically modulates the band edges. For high enough SAW amplitudes, optically generated excitons in the quantum well will be dissociated by the piezoelectric field (inset

*Electronic address: christian.wiele@uni-essen.de of Fig. 1). A field strength of the order of $500 \mathrm{~V} / \mathrm{cm}$ suffices and results in a wave amplitude of 50-150 meV, depending on the wavelength. Carriers are then trapped in the moving lateral potential superlattice of the sound wave and recombination becomes impossible: Electrons will stay in the minima of the wave, while holes move with the maxima [9-11]. A simple estimate of the spatial width $\Delta d$ of the lateral ground state in the wave potential yields $\Delta d / \lambda$ $<0.02$. We thus obtain a series of equally spaced quantum wires moving in the plane of the quantum well. The length of these wires is given by the width of the IDTs, typically 300 $\mu \mathrm{m}$. The occupation of the wires with electrons and holes can be controlled by the pump strength of the laser and is of the order $10^{3}-10^{4}$ carriers per wire.

A quantum dot for our purposes may be established by a stressor on top of the crystal that causes a local potential minimum in the quantum well underneath. The linear dimension of typical stressor dots with transition frequencies in the optical range is about $10-30 \mathrm{~nm}$, while their potential depths are about $100 \mathrm{meV}$ for electrons and $50 \mathrm{meV}$ for holes. For further investigations, we assume that the dot is so small that there is only one electron and one hole level. Occupation of these levels with two carriers of opposite spin is forbidden

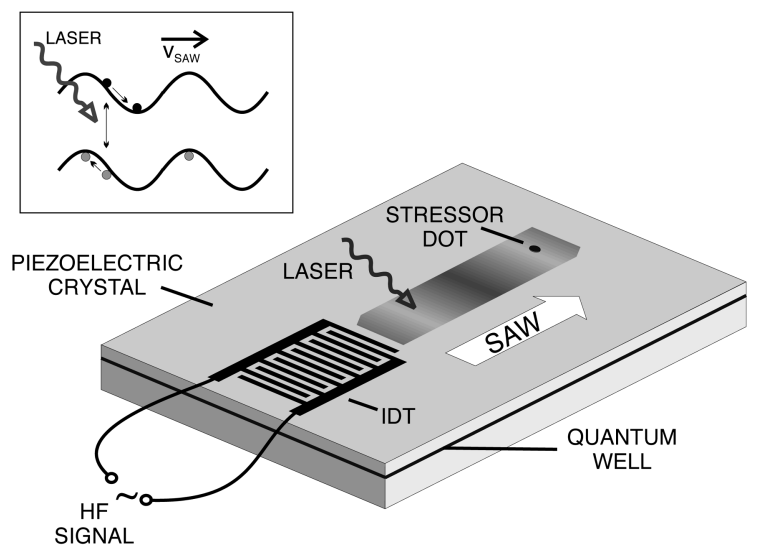

FIG. 1. Schematic sketch of a SAW sample. The material of the system may be, for example, GaAs for the piezoelectric crystal, InGaAs for the quantum well, and InP for the stressor. The inset depicts the storage of optically generated excitons in the potential of the surface acoustic wave. 
by Coulomb blockade. When both levels are occupied an exciton is formed, so there is just a single exciton state. We should speak of an excited, a semiexcited, and an unexcited dot when an exciton, only one carrier (electron or hole), and no carrier are present. An excited or unexcited dot may then be treated as a simple two-level system with pseudospin operators $S^{+}, S^{-}$creating and annihilating an exciton. This system may interact with a single-mode light field. In the semiexcited case no interaction with the light field is possible and the creation of an exciton is only possible by capturing the missing carrier. While being crossed by a moving quantum wire an empty dot may pluck one of the carriers offered: If the dot potential is deep enough a carrier will drop into it and stay there, while the wave is moving on.

The scheme just sketched may indeed produce the designed properties of the pump. First, the periodicity of arriving carriers is given by the SAW, as the moving wires are well separated. Second, with a density of $\approx 3$ carriers per $100 \mathrm{~nm}$ in a wire, there is a high probability for the dot to capture an electron or hole within the crossing time of a wire. Of course, a single dot makes but inefficient use of the moving wires, as only one of $10^{4}$ carriers is used per cycle. If one had several dots lying in a row parallel to the wires, better pump yields could arise. Another way to increase efficiency may be to focus the SAW onto one or a few dots, which seems to be feasible in an experiment.

Our periodically pumped dot (PPD) will in practice suffer from a degradation of complete regularity. One cause of pump fluctuations is the finite width of the lateral SAW ground state, as mentioned above. This leads to variations in the instant of pumping. As indicated above, this will be a few percent of the pump period $T$ given by the SAW frequency. Pump noise also results when no carrier is plucked from a crossing wire; this may be minimized by a high-electron and hole generation rate in the SAW, so all wires are well occupied. For simplicity we neglect both types of noise here and consider the case of zero pump fluctuations. This will allow for a simple analytic description of the process.

For quantum-optical applications we have to couple the PPD to a single-mode light field inside a resonator. As the dot is pumped by a SAW, we cannot put a semiconductor Bragg mirror, which would block the mechanical wave, on top of the device. For the back side this would not be a problem. So we need at least one external mirror for the cavity. The carriers could be generated outside the cavity, as the electrons and holes can be transported over some millimeters by the SAW.

We shall now discuss our pumping scheme in the framework of the Jaynes-Cummings model, limiting ourselves to the single PPD. The system is described by the exciton-field coupling constant $g$, the field damping constant $\kappa$, the pseudospin operators $S^{+}, S^{-}$, and the photon creation and annihilation operators $a^{\dagger}, a$. In the interaction picture the master equation for the density operator of dot and cavity mode is

$$
\begin{aligned}
\dot{\rho} & =g\left[a S^{+}-a^{\dagger} S^{-}, \rho\right]+\frac{\kappa}{2}\left\{\left[a, \rho a^{\dagger}\right]+\left[a \rho, a^{\dagger}\right]\right\} \\
& =: \Lambda \rho .
\end{aligned}
$$

Due to the regularity of pumping events we cannot work with a standard pump term in the master equation. Rather, we have to solve the problem by setting new initial values after every pump event. The Hilbert space is defined in the following way. The dot may be in one of three states, the excited: $|e\rangle$; the unexcited (ground) $|g\rangle$; or the semiexcited $|s e\rangle$, where the special property of the semiexcited state is

$$
S^{+}|s e\rangle=S^{-}|s e\rangle=0,
$$

i.e., a dot in this state cannot interact. The cavity mode is expanded in the basis of Fock states $\mid n)$. We now further assume that every pump event is completely incoherent and destroys all off-diagonal elements of $\rho$. With these assumptions, the most general density operator with the condensed notation $|g, n\rangle:=|g\rangle \mid n)$ is

$$
\begin{aligned}
\rho(t)= & \sum_{n=0}^{\infty}\left[C_{e, e}^{n}(t)|e, n\rangle\left\langle e, n\left|+C_{g, e}^{n}(t)\right| g, n+1\right\rangle\langle e, n|\right. \\
& +C_{e, g}^{n}(t)|e, n\rangle\left\langle g, n+1\left|+C_{g, g}^{n}(t)\right| g, n\right\rangle\langle g, n| \\
& \left.+C_{s e, s e}^{n}(t)|s e, n\rangle\langle s e, n|\right] .
\end{aligned}
$$

Starting from some initial $\rho(0)$ at $t=0$, the system evolves according to the master equation (1) until the first pumping event, immediately before which we have

$$
\rho(T / 2-0)=e^{\Lambda T / 2} \rho(0) .
$$

New initial values at $t=T / 2+0$ are now set by

$$
\begin{gathered}
|s e, n\rangle\langle s e, n|\rightarrow| e, n\rangle\langle e, n|, \\
|g, n\rangle\langle g, n|\rightarrow| s e, n\rangle\langle s e, n|, \\
|e, n\rangle\langle e, n|\rightarrow| e, n\rangle\langle e, n|,
\end{gathered}
$$

all other terms in Eq. (3) vanishing. The three processes indicated have to be interpreted in the following manner: A semiexcited dot is excited by capturing the missing carrier [Eq. (5a)], an unexcited dot captures a carrier and becomes semiexcited [Eq. (5b)], and if the dot is excited at the instant of pumping, no pump event may occur and the system keeps the old state [Eq. (5c)]. We thus find the new initial state after pumping

$$
\begin{aligned}
\rho\left(t_{i}+0\right)= & \sum_{n}\left\{\left[C_{s e, s e}^{n}\left(t_{i}-0\right)+C_{e, e}^{n}\left(t_{i}-0\right)\right]|e, n\rangle\langle e, n|\right. \\
& \left.+C_{g, g}^{n}\left(t_{i}-0\right)|s e, n\rangle\langle s e, n|\right\},
\end{aligned}
$$

$t_{i}=T / 2, T, 3 T / 2 \ldots$ being the instants of pumping. Between the pumping events, the system evolves again like Eq. (4).

\section{PHOTON TRAINS}

As a first application of this new pumping mechanism, a PPD inside a bad single-mode cavity is considered. We thus assume the cavity-damping rate $\kappa$ to be larger than the coupling constant $g$. Let us start with an excited dot and the light field in the vacuum state. The system will then undergo damped Rabi oscillations until the generated photon has left the resonator and the dot is back in the ground state. Now we 
refill the dot with an exciton (first an electron and then a hole) and the process starts again. By doing so, the Hilbert space of the field is confined to the vacuum |0) and the single-photon Fock state $\mid 1$ ), i.e., there is at most one photon in the resonator. With these assumptions Eq. (1) is exactly solvable. In the overdamped case $(4 g<\kappa)$, where a photon is not reabsorbed after emission, we obtain, for the probability of finding a photon in the resonator for a single process,

$$
\begin{aligned}
C_{g, g}^{1}(t) & \equiv p_{1}(t) \\
& =\frac{8 g^{2}}{\kappa^{2}-16 g^{2}} e^{-\kappa t / 2}\left[\cosh \left(\frac{t}{2} \sqrt{\kappa^{2}-16 g^{2}}\right)-1\right] .
\end{aligned}
$$

The long-time behavior of Eq. (7) is

$$
p_{1}(t) \rightarrow e^{-4 g^{2} t / \kappa}
$$

thus the pumping time $T$ has to be much larger than $\kappa / 4 g^{2}$ to ensure that the photon has left the cavity before the next drops into the dot. With these requirements met, the solution for the periodically excited system is

$$
p(t)=\sum_{m=0}^{\infty} p_{1}(t-m T) \Theta[T-|2 t-(2 m+1) T|],
$$

with $\Theta(x)=0$ for $x \leqslant 0$ and 1 for $x>0$. We call this periodic series of one-photon processes a "photon train," having a picture of a long train with equidistant cars in mind. Note particularly that in our system the resonator serves only to enhance the coupling constant $g$ and to orient the emission, not to accumulate photons.

The mean photon number $\bar{n}$ in the cavity is given by the time average of $p(t)$ over one period $T$,

$$
\bar{n}=\frac{1}{T} \int_{0}^{T} d t p(t)=\frac{1}{\kappa T} .
$$

As this system is very simple, most coherence and correlation properties can be calculated analytically. For example, the first-order coherence function $g_{1}(\tau)$ $=\overline{\left\langle a^{\dagger}(t) a(t+\tau)\right\rangle} / \overline{\left\langle a^{\dagger}(t) a(t)\right\rangle}$ yields

$$
g_{1}(\tau)=\sqrt{1+\kappa^{2} / 4 g^{2}} e^{-\kappa|\tau| / 2} \cos (g|\tau|+\phi),
$$

with $\phi=\arctan (\kappa / 2 g)$. A similar quantum-dot device has been proposed in [8] where a different pumping mechanism was considered.

\section{PPD MICROLASER}

The second case to consider is a PPD inside a high- $Q$ single-mode cavity, where photons may be accumulated. We do not want to present a detailed calculation of this model here, as this system does not provide too much that is new. We would rather make a comparison with a standard microlaser model for atoms [12-16].

In the standard model, a beam of regular distributed threelevel atoms goes through an excitation region just before entering a single-mode cavity. Each atom has the probability $p_{A}$ of being excited from its ground level $c$ to the upper level

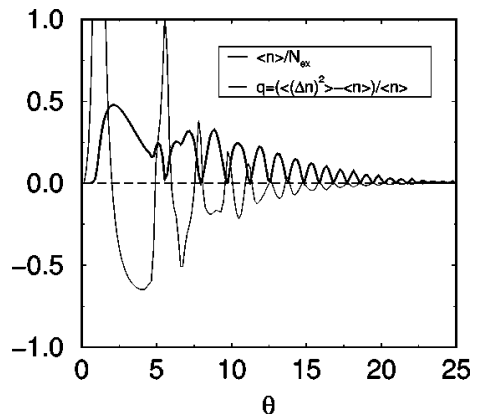

FIG. 2. Numerically calculated stationary photon-number distribution (thick line) and Mendel's $q$ parameter (thin line) for the PPD microlaser. With $N_{e x}=1 /(\kappa T)$ the pump parameter $\Theta$ is defined as $\Theta=\sqrt{N_{e x}} g T / 2$. Here we have $g / \kappa=20$. We find wide ranges of sub-Poissonian distributions $(q<0)$, as known from the atomic microlaser [13].

$a$. The lasing transition involves level $a$ and the intermediate level $b$; thus an atom in level $c$ cannot interact with the light field. Furthermore, it is assumed that at most one atom is in the cavity at a time and that the interaction time $t_{\text {int }}$ is much shorter than the cavity-decay time $\kappa^{-1}$ and the time $T_{A}$ between successive atoms entering the resonator. This assumption allows for neglecting the field damping while the atom passes the cavity, leading to a simple Jaynes-Cummings Hamiltonian during the interaction. In the interval $T_{A}-t_{i n t}$, when no atom is inside the cavity, pure field damping occurs. The parameter $p_{A}$ has been used to describe different pumping statistics [13]. The stationary solution exhibits sub- as well as super-Poissonian statistics, depending on a certain pumping parameter. Another feature of this model is the generation of trapping states in the light field, where the photon number is limited to an upper boundary [16]. This is caused by the constant interaction time $t_{\text {int }}$; as for a specific photon number, the atom leaves the resonator in the excited state (after one or several full Rabi oscillations), and no additional photon is emitted into the cavity.

Now we consider the PPD model, which is also described by the master equation (1), but this time we assume $g$ to be much larger than $\kappa$. Spontaneous emission is again neglected. Starting with a given field, we look at the dot just after a pump event. As described above, the dot is either in the excited or the semiexcited state. This is very much like in the atomic case, where the atom enters the resonator either in the excited level $a$ or in the noninteracting level $c$. From Eq. (6) we may then define the probability $p_{D}\left(t_{i}+0\right)$ of the dot being in the excited state after the $i$ th pump event,

$p_{D}\left(t_{i}+0\right) \equiv \operatorname{Tr}\left(S^{+} S^{-} \rho\right)=\sum_{n=0}^{\infty}\left[C_{s e, s e}^{n}\left(t_{i}-0\right)+C_{e, e}^{n}\left(t_{i}-0\right)\right]$.

As the dot is always inside the cavity, we have $t_{\text {int }} \equiv T / 2$, corresponding to the case of an atom entering the cavity just as the previous one leaves. This circumstance requires the inclusion of field damping during the entire calculation, which for small damping, however, will not lead to essential changes in the results. From Eq. (12) we see that in contrast to the standard microlaser the probability $p_{D}$ of "injecting", an excited dot depends on the state of the "leaving" dot. 
This makes this system much more complicated to treat analytically. But it is clear that $p_{D}$ has to be constant in the stationary regime. The probability has to be determined selfconsistently and is not an independent parameter as in the standard system.

Since both models are very similar, it is not astonishing that we have numerically found all features of the standard microlaser (trapping, sub-, and super-Poissonian statistics) in the PPD model. We give an example of our calculations in Fig. 2. As indicated above, the advantage of the PPD may be a much simpler experimental realization than the atomic microlaser.

\section{CONCLUSION AND OUTLOOK}

We presented the model of a pumping mechanism for semiconductor quantum dots and its applications in quantum optics. The combination of surface acoustic waves, quantum dot physics, and cavities opens an interesting field of research inviting experimental and possibly new theoretical work. Single PPD's offer promise, as indicated above. Collections of several PPD's close by may be put to collective interaction with a single-mode light field. Then one could think of a train of superradiant pulses or a superradiant laser. As our pump mechanism is not only limited to stressor dots, the very homogeneous dots of Nötzel et al. [17] are very promising for such collective effects. Our simplifying assumptions (e.g., a two-level dot) have to be tested in experiments, which may pave the way for a more realistic model.

\section{ACKNOWLEDGMENTS}

One of us (C.W.) would like to thank G. Bastian from the PTB for helpful hints. We further thank J. P. Kotthaus and K. Karrai for helpful discussions.
[1] N. N. Ledentsov, V. A. Shchukin, M. Grundmann, N. Kirstaedter, J. Böhrer, O. Schmidt, D. Bimberg, V. M. Ustinov, A. Yu. Egorov, A. E. Zhukov, P. S. Kop'ev, S. V. Zaitsev, N. Yu. Gordeev, Zh. I. Alferov, A. I. Borovkov, A. O. Kosogov, S. S. Ruvimov, P. Werner, U. Gösele, and J. Heydenreich, Phys. Rev. B 54, 8743 (1996).

[2] N. Kirstaedter, O. G. Schmidt, N. N. Ledentsov, D. Bimberg, V. M. Ustinov, A. Yu. Egorov, A. E. Zhukov, M. V. Maximov, P. S. Kop'ev, and Zh. I. Alferov, Appl. Phys. Lett. 69, 1226 (1996).

[3] M. Grundmann and D. Bimberg, Phys. Status Solidi A 164, 279 (1997).

[4] M. Asada, Y. Miyamoto, and Y. Suematsu, IEEE J. Quantum Electron. 22, 1915 (1986).

[5] H. Saito, K. Nishi, I. Ogura, S. Sugou, and Y. Sugimoto, Appl. Phys. Lett. 69, 3140 (1996).

[6] H. Shoji, Y. Nakata, K. Mukai, Y. Sugiyama, M. Sugawara, N. Yokoyama, and H. Ishikawa, Jpn. J. Appl. Phys., Part 1 35, 903 (1996).

[7] J. A. Lott, N. N Ledentsov, V. M. Ustinov, A. Yu. Egorov, A. E. Zhukov, P. S. Kop'ev, Zh. I. Alferov, and D. Bimberg, Electron. Lett. 33, 1150 (1997).

[8] A. Imamoḡlu and Y. Yamamoto, Phys. Rev. Lett. 72, 210 (1994).
[9] C. Rocke, S. Zimmermann, A. Wixforth, J. P. Kotthaus, G. Böhm, and G. Weimann, Phys. Rev. Lett. 78, 4099 (1997); e-print cond-mat/9704029.

[10] C. Rocke, A. Wixforth, J. P. Kotthaus, W. Klein, H. Böhm, and G. Weimann, Inst. Phys. Conf. Ser. 155, 125 (1997); eprint cond-mat/9609250.

[11] J. M. Shilton, V. I. Talyanskii, M. Pepper, D. A. Ritchie, J. E. F. Frost, C. J. B. Ford, C. G. Smith, and G. A. C. Jones, J. Phys.: Condens. Matter 8, 531 (1996).

[12] P. Filipowicz, J. Javanainen, and P. Meystre, Phys. Rev. A 34, 3077 (1986).

[13] J. Bergou, L. Davidovich, M. Orszag, C. Benkert, M. Hillery, and M. O. Scully, Phys. Rev. A 40, 5073 (1989).

[14] F. Haake, S. M. Tan, and D. F. Walls, Phys. Rev. A 40, 7121 (1989).

[15] S. Y. Zhu, M. S. Zubairy, C. Su, and J. Bergou, Phys. Rev. A 45, 499 (1992).

[16] R. R. Puri, F. Haake, and D. Forster, J. Opt. Soc. Am. B 13, 2689 (1996).

[17] R. Nötzel, Z. Niu, M. Ramsteiner, H.-P. Schönherr, A. Tranpert, L. Dräweritz, and K. H. Ploog, Nature (London) 392, 56 (1998). 